\title{
Le vocabulaire et la rhétorique des comptabilités médiévales. Modèles, innovations, formalisation
}

Dijon-Besançon, table ronde, 30 septembre- $1^{\mathrm{er}}$ octobre 2010

\section{Patrice Beck}

\section{(2) OpenEdition}

\section{Journals}

Édition électronique

URL : https://journals.openedition.org/cem/11578

DOI : $10.4000 /$ cem. 11578

ISSN : 1954-3093

Éditeur

Centre d'études médiévales Saint-Germain d'Auxerre

Édition imprimée

Pagination : 267-271

ISSN : 1623-5770

\section{Référence électronique}

Patrice Beck, «Le vocabulaire et la rhétorique des comptabilités médiévales. Modèles, innovations,

formalisation », Bulletin du centre d'études médiévales d'Auxerre | BUCEMA [En ligne], 14 | 2010, mis en ligne le 14 octobre 2010, consulté le 22 septembre 2022. URL : http://journals.openedition.org/cem/ 11578 ; DOI : https://doi.org/10.4000/cem.11578

Ce document a été généré automatiquement le 22 septembre 2022.

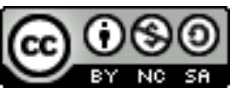

Creative Commons - Attribution - Pas d'Utilisation Commerciale - Partage dans les Mêmes Conditions 4.0 International - CC BY-NC-SA 4.0

https://creativecommons.org/licenses/by-nc-sa/4.0/ 


\section{Le vocabulaire et la rhétorique des comptabilités médiévales. Modèles, innovations, formalisation}

Dijon-Besançon, table ronde, 30 septembre- $1^{\text {er }}$ octobre 2010

\section{Patrice Beck}

1 Cette table ronde constitue la troisième partie d'une enquête collective 2008-2012 sur les «Comptabilités et comptables à la fin du Moyen Âge. Codicologie, diplomatique et prosopographie» coordonnée par Patrice Beck (IRHiS, université Charles de Gaulle Lille 3).

2 L'importance des archives comptables médiévales conservées en Europe est bien connue: leur contenu a suscité et continue de nourrir nombre d'études d'histoire sociale, économique ou institutionnelle, d'histoire agraire, urbaine ou des techniques. Mais aucune étude codicologique et diplomatique précise et d'envergure, c'est-à-dire d'analyse détaillée des conditions spécifiques de leur fabrication et de leurs usages, n'en a été jusqu'à présent tentée, en France comme ailleurs en Europe. Les historiens sont naturellement plus intéressés par les contenus socio-économiques et les spécialistes de l'analyse formelle et matérielle des manuscrits, même ceux qui militent depuis les années 1980 en faveur d'une «codicologie quantitative» appliquée aux séries, ont surtout porté attention aux livres des bibliothèques et aux chartes, comme supports de production et de diffusion culturelle, plutôt qu'aux registres de gestion ${ }^{1}$.

3 S'attacher à l'analyse de la nature et du format du support d'écriture, du nombre et de l'organisation des feuillets et des cahiers, de la réglure et de la mise en page, de l'écriture et de l'organisation du texte, des mots employés et des valeurs affichées dans les documents administratifs conservés en séries bien datées et localisées, présente cependant quelque intérêt: celui de donner des arguments chiffrés pour discuter à la fois des buts poursuivis par l'administration émettrice et des compétences de ses agents. Y a-t-il des normes communes, sont-elles partout appliquées, comment évoluent-elles et comment en contrôle-t-on l'application? Quelles sont les manières d'identifier, de classer et de compter qui sont alors utilisées pour gérer le temps, les 
biens et les personnes? Comment donc et par qui les princes et les seigneurs, les édiles et les marchands, les moines et les chanoines font-ils tenir leurs comptes, en Europe occidentale aux temps de la «genèse médiévale» des États modernes?

4 Tel est le programme qu'un groupe de chercheurs a entrepris de développer sous quatre formes:

- animation d'un espace de travail et d'échange ouvert à tous à l'adresse électronique suivante: http://irhis.recherche.univ-lille3.fr/00-Comptabilites/Index.html;

- participation au développement d'une publication électronique nommée Revue d'histoire des comptabilités, en cours de labellisation auprès de Revues.org;

- organisation d'une série de tables rondes, dont les actes seront publiés dans la susdite revue;

- organisation en 2012 d'un colloque international élargissant le champ des interventions et des intervenants.

5 En 2008, la réunion fondatrice de Lille a mis au point une grille générale d'analyse:

\begin{tabular}{|c|c|c|}
\hline SUPPORT & $\begin{array}{l}\text { pappros - cire - ardoise - parchemin - papir } \\
\text { filigrane, vergeure, pontuse anx }\end{array}$ & $\rightarrow$ fabrication - commerc is lisation \\
\hline FORIMAT \& COMPOSITION & $\begin{array}{l}\text { feuillet -rouleau -registre } \\
\mathrm{r}^{\circ} \& \mathrm{v}^{\prime} \text { - bifeuillt - cahier } \\
\text { courerture \& re lime }\end{array}$ & usages - archivage \\
\hline MISE EN FORME & $\begin{array}{l}\text { gestion des défauts - côté poil, côté chair des parchemins } \\
\text { homogénéité du papier (filigranes) }\end{array}$ & esthétique \\
\hline REGLURE \& MISE EN PAGE & $\begin{array}{l}\text { poirte sèche et grasse, plinire } \\
\text { colorine }(s) \& \text { marge (s) } \\
\text { fo liotation }\end{array}$ & $\begin{array}{l}\rightarrow \text { esthétique } \\
\rightarrow \text { stritégies administratives }\end{array}$ \\
\hline ECRITURE \& SIGHATURE & $\begin{array}{l}\text { les mains et kur emplac emert (gaphobgi) } \\
\text { signatirles (parshobgie) }\end{array}$ & $\rightarrow$ formation des interverarts \\
\hline $\begin{array}{l}\text { PREAMBULE \& ESCHA TOCOLE } \\
\text { ANHOTATION(s) }\end{array}$ & $\begin{array}{l}\text { les drectives, les normes et kur spplication } \\
\text { le contrôle }\end{array}$ & $\begin{array}{l}\rightarrow \text { compétences des interverarts } \\
\rightarrow \text { efficacité de l'administration }\end{array}$ \\
\hline ORGAHISATION DU PROPOS & ordie de présertation, titres, hiérarchisation & $\rightarrow$ bzique administrative, enjeux \\
\hline VOCABULAIRE & substantifs, qua lificatifs, verbes & $\rightarrow$ k riétorique dupounor et sa réception \\
\hline QUANTOMETRIE & dates - chiffires et rombres - opérations & $\rightarrow$ compétences et rizueur des squibes \\
\hline
\end{tabular}

\section{Table ronde $n^{\circ} 2$ : Codicologie des documents comptables}

6 En octobre 2009, la réunion de Paris, co-organisée par les laboratoires LAMOP (université de Paris 1 Panthéon Sorbonne) et IRHiS (université de Lille 3), a abordé les aspects formels des comptabilités.

7 Jeudi $1^{\text {er }}$ et vendredi 2 octobre 2009, université de Paris 1 Sorbonne, salle Perroy.

- P. BECK et O. MATTÉONI, Introduction

- P. BERTRAND (IRHT, Orléans), Codicologie comparée des censiers et des comptabilités dans le domaine français aux XIII et XIV siècles

- M.-L. JALABERT (université de Paris 1), L'écriture comptable des bayles des archevêques de Narbonne Bernard de Fargues (1311-1314) et Gaubert du Val (1341-1347)

- C. JÉHANNo (université du Littoral), Les aspects codicologiques de la série des comptes de l'Hôtel-Dieu de Paris à la fin du Moyen Âge (dernier quart du XIVe-début du XVI ${ }^{e}$ siècle) 
-S. BEPOIX (université de Franche-Comté), F. couvel (université de Paris 1) et M. LEGUiL (université de Franche-Comté), La fabrique des comptes des châtellenies dans les duché et comté de Bourgogne aux XIV et $X V^{e}$ siècles: entre exercice imposé et particularismes locaux

-J.-B. SANTAMARIA (université de Lille 3), Ruptures politiques et mutations comptables au bailliage d'Hesdin en Artois au XIV siècle

- F. DUCEPPe-lamarRe (université de Lille 3), Copier des comptes à l'époque de Mahaut d'Artois

- H. DEWEZ (université de Paris 1), Le rouleau comme support des comptes manoriaux au prieuré cathédral de Norwich (mi XIII'-mi XIVe siècle)

- D. ANGERS (université de Montréal, Canada), Codicologie des comptabilités de la vicomté de Caen à la fin du Moyen Âge

- E. GRÉLOIS (université de Rennes 2), L'usage du papier en Basse-Auvergne (ca 1250-ca 1320). Autour de trois comptabilités conservées des consuls de Montferrand (1259-1319), des bénédictines de Beaumont (1294-1311) et de l'évêché de Clermont (1316-1319)

- P. ВЕСК (université de Lille 3), Les comptabilités de la commune de Dijon

- melo (université de Minho, Portugal), Comptabilités municipales: les livres des comptes de Porto dans la deuxième moitié $\mathrm{du} \mathrm{XV}^{e}$ siècle

\section{Table ronde $n^{\circ} 3$ : Le vocabulaire et la rhétorique des comptabilités médiévales. Modèles, innovations, formalisation.}

8 Les 30 septembre et $1^{\mathrm{er}}$ octobre 2010, la troisième rencontre est organisée à Dijon (Archives municipales) et à Besançon (université de Franche-Comté). Elle aura pour thème le vocabulaire comptable.

9 Comité d'organisation et institutions partenaires: Patrice Beck (IRHiS, université de Lille 3), Sylvie Bepoix (Laboratoire de sciences historiques, université de FrancheComté), Éliane Lochot (Archives municipales de Dijon), Eliana Magnani (CNRS, ARTeHIS), Olivier Mattéoni (LAMOP, université de Paris 1) et Daniel Russo (ARTeHIS, université de Bourgogne).

Il s'agit de révéler les manières dont les institutions comptables et leurs agents ordonnent, justifient, décrivent, présentent et se représentent leur activité. Seront interrogés les ordonnances portant création des Chambres des comptes et les lettres de commission instituant aux offices, comme les comptabilités elles-mêmes, qui, dans leurs préambules comme dans leurs arrêts, dans leurs titres comme dans l'organisation de l'information, énoncent des choix et donc les valeurs qui soustendent l'activité. Afin d'alimenter les études comparatistes, seront particulièrement étudiés la désignation des comptes et des objets, l'ordre de présentation des rubriques, le vocabulaire de l'autorité comptable, les titres des institutions et des agents comptables, les compétences et les vertus requises et reconnues des agents comptables, les termes et les signes techniques de validation et de contrôle.

11 Rappel du programme

12 Jeudi 30 septembre 2010 (Dijon, Hôtel de ville, cour de Flore, Salon Porte aux Lions), $9 \mathrm{~h} 30-18 \mathrm{~h} 00$

- P. ВECK (université de Lille 3), Introduction 
- WIRTH (université catholique de Louvain, Belgique), La rhétorique des comptabilités lorraines: l'exemple des comptes du receveur de Châtel-sur-Moselle (1431-1507)

-J.-M. YANTE (université catholique de Louvain, Belgique), Des comptabilités luxembourgeoises de la fin $d u X^{X} v^{e}$ siècle aux comptabilités bourguignonnes en Luxembourg au milieu du Xvesiècle. Permanences et mutations

-D. ANGERs (université de Montréal, Canada), Le vocabulaire des comptabilités normandes: l'exemple des vicomtés de Caen et d'Auge

- THEILler (université de Toulouse 2 Le Mirail), Le vocabulaire des comptes et des comptables en Normandie orientale entre XIV ${ }^{e}$ et $X V I^{e}$ siècles

- O. MATtÉoni (université de Paris 1 Panthéon Sorbonne), Mots, langue et discours dans les comptes foréziens de la fin du Moyen Âge

- SCHNERB (université de Lille 3), Le vocabulaire des comptabilités de guerre dans l'espace francobourguignon à la fin du Moyen Âge

- Russo (université de Bourgogne, ARTeHIS), «Ars et ratio». Écrire, composer et voir les «grands joyaux» au temps de Philippe le Hardi

-S. Jolivet (ARTeHIS), Justifier les dépenses vestimentaires dans la recette générale de toutes les finances du duc Philippe le Bon

13 Visite aux Archives municipales de Dijon (É. Lоснот, conservatrice)

14 Vendredi $1^{\mathrm{er}}$ octobre 2010 (Besançon, faculté des Lettres, rue Mégevand, grand Salon), 9 h00 - 17 h00

-S. Bepoix (université de Franche-Comté) \& F. couvel (université de Paris 1 PanthéonSorbonne), Rendre bon compte en Bourgogne à la fin du Moyen Âge: le dire au travers des ordonnances et le faire selon les mots des receveurs

- F. DUCEPPE-LAMARRE (université de Lille 3), Le vocabulaire de la copie: les doublons de comptes de la comtesse Mahaut d'Artois (début du XIV siècle)

- M. AUBRy (université de Lille 3), Le comptable au travail: les signes techniques en marge des comptabilités lilloises (1291-1369)

- Melo (université de Minho, Portugal), Le vocabulaire des comptabilités portugaises des XIVe et $\mathrm{XV}$ siècles

- M.-L. JALABERT (université de Paris 1 Panthéon Sorbonne), Des «comptes à régler» avec les archevêques de Narbonne au milieu du XIve siècle

- G. PINTo (université de Florence, Italie), Considérations sur la comptabilité des hôpitaux de la Toscane des XIV et XVe siècles

- L.-H. Gouffran (université de Provence), L'écriture comptable d'un marchand provençal au tournant du XVe siècle: les comptabilités de Bertrand de Roquefort

- M. LEGUIL (université de Franche-Comté), Livre, papier des comptes, compte particulier: les documents de gestion privée des officiers de finance bourguignons aux XIV et XV siècles

Discussion finale, projets

Contact: patrice.beck3@orange.fr 


\section{NOTES}

1. C. Bozzolo et E. ornato, Pour une histoire du livre au Moyen Âge. Trois essais de codicologie quantitative, Paris, 1980 ( $2^{\mathrm{e}}$ éd. 1983) ; A. DEROLEZ, Codicologie des manuscrits en écriture humanistique sur parchemin, Turnhout, 1984; D. MUZERELLE, Vocabulaire codicologique, répertoire méthodique des termes français relatifs aux manuscrits, Paris, 1985; M. ORNATO et N. PONS (dir.), Pratique de la culture écrite en France au Xve siècle, Louvain-la-Neuve, 1995. Sur les 350 notices contenant le volume $2 \mathrm{du}$ Bulletin codicologique édité en 2005 par le Centre international de codicologie de Bruxelles, aucune ne porte sur les actes de la pratique comptable.

\section{INDEX}

Mots-clés : comptabilité, vocabulaire 